\title{
Endoscopic Endonasal Resection of Sinonasal and Nasopharyngeal Pleomorphic Adenomas: A Case Series
}

\author{
Case Report Apostolos Karligkiotis ${ }^{1}$ (D), Gülpembe Bozkurt², 3 (D), Giacomo Pietrobon ${ }^{4}$ (D), Paolo Battaglia ${ }^{2,3}$ (D),

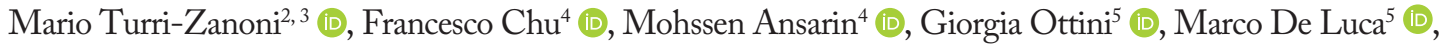 \\ Silvia Uccella ${ }^{5}$ (D), Paolo Castelnuovo ${ }^{2,3}$ (D) \\ ${ }^{1}$ Division of Otorhinolaryngology, Department of Surgical Specialties, ASST Sette Laghi, Ospedale di Circolo e Fondazione \\ Macchi, Varese, Italy \\ ${ }^{2}$ Division of Otorhinolaryngology, Department of Biotechnology and Life Sciences, University of Insubria-Varese, ASST Sette \\ Laghi, Ospedale di Circolo e Fondazione Macchi, Varese, Italy \\ ${ }^{3}$ Department of Biotechnology and Life Sciences, Head and Neck Surgery \& Forensic Dissection Research Center (HNS\&FDRc), \\ University of Insubria, Varese, Italy \\ ${ }^{4}$ Division of Otolaryngology and Head and Neck Surgery, European Institute of Oncology IRCCS, Milan, Italy \\ ${ }^{5}$ Division of Pathology, Department of Medicine and Surgery, University of Insubria, ASST Sette Laghi, Ospedale di Circolo e \\ Fondazione Macchi, Varese, Italy
}

\begin{abstract}
The aim of this study is to describe the clinicopathological characteristics of intranasal pleomorphic adenomas (PAs), as well as the role and outcomes of endoscopic endonasal resection. A retrospective review of the clinical data from patients with PA of the nasal cavity who were treated by the authors at three tertiary medical centers between June 1998 and December 2019. A total of five patients with PA were found. Three patients were male, two were female and their mean age was 62.2 years. All cases were resected "en bloc" with endoscopic endonasal approach. No evidence of disease
\end{abstract}

ORCID iDs of the authors:

A.K. 0000-0003-0129-9908;

G.B. 0000-0003-2100-023X;

G.P. 0000-0003-4981-1851;

P.B. 0000-0001-6189-5889;

M.T.Z. 0000-0002-3678-9088;

F.C. 0000-0003-4813-1475;

M.A. 0000-0002-8384-5388;

G.0.0000-0003-0068-0050;

M.D.L. 0000-0002-5522-4496;

S.U. 0000-0002-5714-7295;

P.C. 0000-0002-5184-6140.

Cite this article as: Karligkiotis A, Bozkurt G, Pietrobon G, Battaglia P, Turri-Zanoni M, Chu F, et al. Endoscopic Endonasal Resection of Sinonasal and Nasopharyngeal Pleomorphic

Adenomas: A Case Series. Turk Arch

Otorhinolaryngol 2020; 58(3): 186-92.

\section{Corresponding Author:}

Gülpembe Bozkurt; gptalayhan@gmail.com

Received Date: 13.01.2020

Accepted Date: 12.07.2020

Content of this journal is licensed under a Creative Commons Attribution 4.0 International License.

Available online at www.turkarchotolaryngol.net

\section{Introduction}

Pleomorphic adenoma (PA) is the most common benign tumor of the salivary glands. It is a benign, epithelial-derived tumor, demonstrating both epithelial and mesenchymal differentiation. Its histopathological diagnosis is usually straightforward, but especially on small diagnostic biopsies, it can require the employment of second level ancillary techniques, such as immunohistochemistry. Besides the typical variant of $\mathrm{PA}$, cellular and atypical variants are recognized, and immunostaining for both the epithelial (cytokeratins, EMA, CEA, CD117) and stromal components (calponin, S100 protein, glial fibrillary acidic protein, actin, vimentin) may be useful to characterize the tumor. In addition, a relatively new marker, the PA gene 1 (PLAG1) has been reported to be consistently positive in the nuclei of epithelial and stromal cells. Also, molecular genetics can be helpful in diagnosing difficult cases, as $70 \%$ of PAs are karyotypically abnormal, with rearrangements of chromosome 8q12 (in the region of PLAG1), including trans- was observed during a mean follow-up of 10.6 years. No case presented with malignant transformation into carcinoma ex-PA. PA of the sinonasal tract and the nasopharynx is difficult to diagnose due to nonspecific clinical and radiological findings. Endoscopic endonasal approaches can be considered the gold standard in the treatment of these tumors and provide excellent visual control of the surgical field and clear margins.

Keywords: Paranasal sinus, nasal cavity, benign tumor, endoscopic surgical procedure, salivary gland, pleomorphic adenoma locations $\mathrm{t}(3 ; 8)(\mathrm{p} 21 ; \mathrm{q} 12)$ and $\mathrm{t}(5 ; 8)(\mathrm{p} 13 ; \mathrm{q} 12)$; and of chromosome 12q14-15 (in the region of high mobility group AT-hook 2 gene HMGA2), including translocation $\mathrm{t}(9 ; 12)(\mathrm{p} 24 ; \mathrm{q} 14-15)$ or ins $(9 ; 12)(\mathrm{p} 24 ; \mathrm{q} 12 \mathrm{q} 15)(1)$.

Although PA mainly arises from the major salivary glands, especially in the parotid, involvement of the upper aerodigestive tract has been described in the literature (2). Intranasal localization is extremely rare and approximately less than 120 cases arising from the nasal septum, the lateral nasal wall and the nasopharynx have been reported (2, $3)$. The treatment of choice is surgical resection with clear margins and several surgical approaches have been proposed including intranasal resection, lateral rhinotomy and midface degloving. In the recent years, improvement in anatomical knowledge, imaging techniques, surgical skills and computer-aided surgery have led endoscopic endonasal procedures to be feasible and advisable for the management of benign and malignant sinonasal, skull base and nasopharyngeal lesions. Yet, only 
limited (approximately 50) endoscopically-treated cases have been reported because of the rarity of pleomorphic adenoma within the nasal cavity $(2,3)$.

In this paper we describe our experience with five cases of intranasal pleomorphic adenoma focusing on the clinicopathological characteristics, as well as on the role and outcomes after endoscopic endonasal resection.

A total of five patients who were treated by the same surgical team for sinonasal pleomorphic adenoma in the Departments of Otorhinolaryngology of the University Hospitals of Varese and Pavia and the Division of Otolaryngology and Head and Neck Surgery of the European Institute of Oncology in Milan, between June 1998 and December 2019. Data related to patient demographics were retrieved from a specific database. Patients with a minimum follow-up period of 24 months were included in the study (Table 1). The study received approval from the Board of Ethics of Ospedale di Circolo e Fondazione Machi (Approval Date: July 7, 2015; Approval Number: 0033025).

Nasal endoscopy and imaging were utilized for assessing the extension of the tumor. Computed tomography (CT) and/or magnetic resonance imaging (MRI) were performed in all patients to better evaluate tumor extension and to differentiate inflammatory from neoplastic involvement of the sinuses. Following the evaluation by imaging, diagnostic biopsy (when feasible due to anatomical factors) was performed under local or general anesthesia by endoscopic examination.

All patients underwent endoscopic endonasal resection under general anesthesia. They were fully informed about the methods of treatment and gave their written consent. The surgical specimen was sent to the Pathology Department for evaluation. Formalin-fixed and paraffin-embedded samples of the lesions were microscopically examined using hematoxylin and eosin-stained slides, and when needed, immunohistochemical analysis using routine protocols.

In the postoperative period, we followed up the patients with periodic endoscopic examinations. Lack of visualization of the primary site involved, presenting scar tissue or suspected recur-

\section{Main Points}

- PAs of the sinonasal tract and the nasopharynx are difficult to diagnose due to nonspecific clinical and radiological findings.

- Accurate histopathological examination by an expert head and neck pathologist is needed to avoid preoperative misdiagnosis.

- Endoscopic endonasal "en bloc" approaches can be considered the gold standard for the treatment of these tumors with excellent visual control of the surgical field and clear margins.

- In case of large-sized tumors, a progressive disassembling of the lesion ("piecemeal" resection) can also be employed by means of endoscopic visualization, keeping in view the limits between normal and diseased mucosa. rence, required postoperative CT scanning and/or MRI and a biopsy under local anesthesia in the postoperative protocol.

\section{Case Presentation}

\section{Case 1}

A 71-year-old woman presented with progressive left nasal obstruction that had developed over the years and a single episode of epistaxis. A large, pink-grey polypoid mass, occupying the left nasal cavity was detected in nasal endoscopy. The large tumor size prevented the visualization of the olfactory cleft and of the osteomeatal complex, as well as of the site of origin of the lesion. The overlying mucosa was intact and no cervical lymphadenopathy was detected. CT scan showed a left lesion originating from the left lateral nasal wall and involving the medial wall of nasolacrimal duct and the nasal vestibule. The olfactory cleft and the paranasal sinuses were not involved. Iodine contrast failed to show homogeneous enhancement but excluded the characteristics of a vascular lesion. A diagnostic biopsy was performed, and based on the histopathological diagnosis of $\mathrm{PA}$, the patient underwent an endoscopic endonasal approach with "en bloc" subperiosteal excision. Surgical procedure included the resection of the middle turbinate by sparing its insertion in the skull base, middle meatal antrostomy, and resection of the ethmoid bulla with clear margins at frozen section. Perioperatively, the lesion was seen to originate from the lateral nasal wall and extend towards the nasal septum without infiltrating it. A complete resection was confirmed in the final histopathological report. Three years later the patient experienced a left fronto-ethmoidal mucocele that was successfully marsupialized endoscopically. No evidence of relapse of the pathology was observed in the eight years after surgery, however the patient stopped coming to her follow-up visits.

\section{Case 2}

A 50-year-old man was referred with progressive bilateral nasal obstruction that had developed during the previous year and recent external nasal deformation. Nasal endoscopy revealed a mass occupying the right nasal cavity, covered by intact mucosa and displacing the nasal septum to the left side. No cervical lymphadenopathy was observed. CT scan showed a median lesion with homogeneous density, displacing the nasal septum bilaterally without bone or cartilage infiltration. The paranasal sinuses were not involved. MRI could not be performed because the patient was claustrophobic. Biopsy revealed that the lesion was a PA. Under general anaesthesia, an endoscopic endonasal approach with "en bloc" excision of the mass and the nasal septum was performed. After 18 years of follow-up, nasal endoscopy and CT scan demonstrated no residual disease.

\section{Case 3}

A 57-year-old man presented with a history of left recurrent epistaxis and nasal obstruction that worsened during the previous month. Nasal endoscopy showed a hemorrhagic polypoid mass filling the anterior part of the left nasal cavity with a right-sided septal deviation. The nasopharynx was intact and no neck lymphadenopathy was observed on physical examination. $\mathrm{CT}$ and MRI revealed a homogenously enhancing large lobular 
mass in the left nasal cavity, involving the medial wall of the maxillary sinus (which was thinned) and the inferior turbinate. MRI revealed a multilobular lesion extending from the inferior turbinate, the piriform crest, the lateral nasal wall to the septum. The wall of the nasolacrimal duct was partially not evident and the septum seemed to be not involved by the tumor. Putative diagnosis based on preoperative assessment was nasal hemangioma. Biopsy of the nasal lesion showed biphasic proliferation of epithelial cells that contained polygonal and mucus holding elements with mild nuclear atypia. Stromal component was poorly represented. The histopathological report on this sample was consistent with a low grade mucoepidermoid carcinoma. The patient underwent staging for regional or distant metastasis that was negative and subsequent endoscopic endonasal "en bloc" resection with left radical ethmoidal sphenoidectomy, medial maxillectomy with nasolacrimal duct sectioning, frontal sinusotomy and removal of the septum. Multiple frozen sections were performed intraoperatively and the medial maxillary wall was identified as the site of origin of the neoplasm. The whole surgical specimen of the neoplasm was sent to histopathological examination where microscopic picture showed a mixture of mesenchymal and epithelial elements. The former had spindle cell differentiation and focal areas with chondroid or myxoid matrix with plumped cells. The latter showed polygonal aspects with squamous differentiation, as well as mucous secreting aspects seen in the preoperative sample. These morphological aspects were confirmed with immunostaining for cytokeratins, CEA, p63, S100 and vimentin and the final pathology report was PA with focal squamous and muciparous differentiation (Figure $1 \mathrm{a}$ and $1 \mathrm{~b}$ ). One year after the surgery the patient experienced post-saccal lacrimal pathway obstruction that was treated by endonasal dacryocystorhinostomy. The patient suffered a stroke with significant cognitive impairment three years ago and is currently free of the disease 13 years after surgery.

\section{Case 4}

A 75-year-old man was referred from another institution with a history of right hearing loss ongoing for several years. He underwent contrast enhanced $\mathrm{CT}$ scan with evidence of a 13 $\mathrm{mm}$ expansive lesion at the posterior border of the right torus in the Rosenmüller's fossa, with calcified foci inside and bright homogeneous enhancement. No bone erosion or enlarged locoregional lymph nodes were detected. MR imaging, which was performed two months later, revealed a $16 \mathrm{~mm}$ heterogeneous and irregularly enhanced right nasopharyngeal mass, without bone erosion (Figure 2). Subsequent biopsy of the lesion was interpreted by the local pathologists as an extracranial craniopharyngioma. The patient was then transferred to our department where nasopharyngeal endoscopic examination showed a mucosal swelling at the right postero-lateral nasopharyngeal wall. The original slides of the previous biopsies were reviewed in our pathology department and the possibility of PA was suggested. The patient underwent a type 1 nasopharyngeal "en bloc" endoscopic resection as described by Castelnuovo et al. (4). No ventilation tube was inserted in the right tympanic membrane. Definitive histologic examinations with immunohistochemical studies confirmed the diagnosis of PA (Figure 1c and 1d). The patient is currently free of disease 12 years after the surgery and no Eustachian tube dysfunction has been observed.

\section{Case 5}

A 58-year-old woman was referred from another institution because of the incidental finding of a gadolinium enhanced lesion (24x18 mm) of the left nasolacrimal duct during an MRI for the regular follow-up of Neurofibromatosis type 1. She presented onset of epiphora of the left eye in the previous months. History of multiple resections of cutaneous fibromas of the left maxillary region was present, as well. Nasal endoscopy revealed a submucosal swelling of the head of the left turbinate. She underwent a paranasal sinus CT scan which showed bony deformation of the medial wall of the maxillary sinus and the inferior turbinate without signs of bony erosion/infiltration (Figure 3). A biopsy of the lesion was taken under general anesthesia with endoscopic endonasal approach. Histopathologic examination was compatible with eosinophilic adenoma (oncocytoma). She was then submitted to a left transnasal endoscopic partial maxillectomy (type

Table 1. Summary of clinical and surgical findings in relation to the follow-up status of the patients included in this analysis

\begin{tabular}{|c|c|c|c|c|c|c|c|c|c|c|c|}
\hline Patient $^{\#}$ & $\begin{array}{c}\text { Age, } \\
\text { years/Sex }\end{array}$ & Side & Site & Symptoms & $\begin{array}{c}\text { Preoperative } \\
\text { diagnosis }\end{array}$ & $\begin{array}{l}\text { Surgical } \\
\text { approach }\end{array}$ & $\begin{array}{l}\text { Surgical } \\
\text { manner }\end{array}$ & $\begin{array}{c}\text { Final } \\
\text { pathology }\end{array}$ & $\begin{array}{l}\text { Postoperative } \\
\text { complications }\end{array}$ & $\begin{array}{l}\text { Clinical } \\
\text { outcome }\end{array}$ & $\begin{array}{l}\text { Follow-up period } \\
\text { (months) }\end{array}$ \\
\hline 1 & $71 / \mathrm{F}$ & $\mathrm{L}$ & LNW & NO, Ex & $\mathrm{PA}$ & EER & En bloc & $\mathrm{PA}$ & Mucocele (late) & NED & $\begin{array}{l}96 \text { (then lost to } \\
\text { follow up) }\end{array}$ \\
\hline 2 & $50 / \mathrm{M}$ & $\mathrm{R}$ & S & $\mathrm{NO}$ & $\mathrm{PA}$ & EER & En bloc & $\mathrm{PA}$ & - & NED & 216 \\
\hline 3 & $57 / \mathrm{M}$ & $\mathrm{L}$ & LNW & Ex, NO & LGMEC & EER & En bloc & $\mathrm{PA}$ & $\begin{array}{l}\text { Post-saccal } \\
\text { lacrimal } \\
\text { pathway } \\
\text { obstruction } \\
\text { (late) }\end{array}$ & NED & 156 \\
\hline 4 & $75 / \mathrm{M}$ & $\mathrm{R}$ & $\mathrm{RF}$ & HL & $\mathrm{ExCCP}$ & EER & En bloc & $\mathrm{PA}$ & - & NED & 144 \\
\hline 5 & $58 / \mathrm{F}$ & $\mathrm{L}$ & LNW & Ep & $\mathrm{PA}$ & EER & En bloc & $\mathrm{PA}$ & - & NED & 25 \\
\hline
\end{tabular}



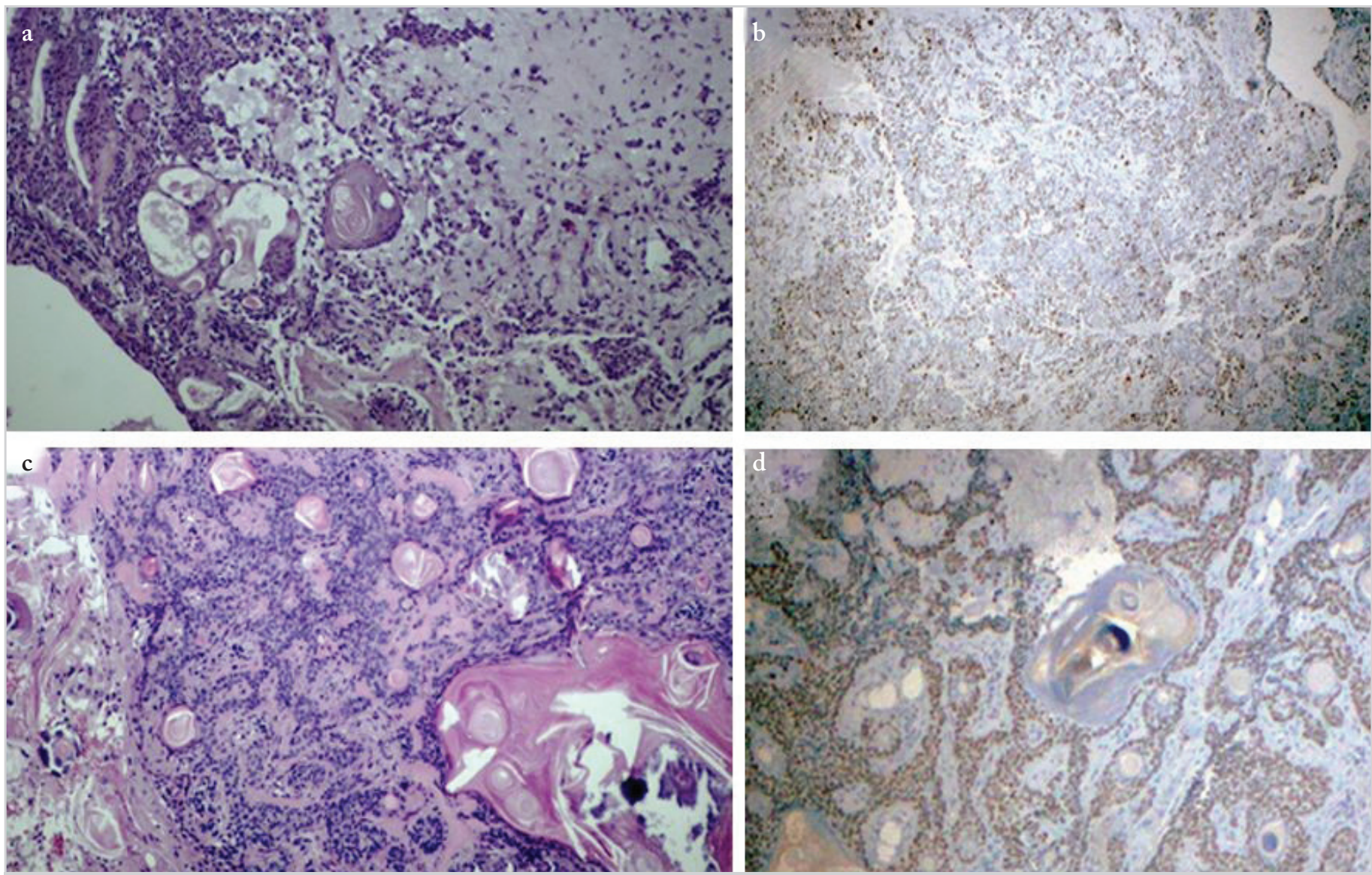

Figure 1. a-d. Pleomorphic Adenomas. Case 3. (a) Squamoid and mucus producing cells with pale cytoplasm and peripherally displaced nuclei and cells of intermediate type with keratinization (hematoxylin-eosin stain, original magnification x20). (b) Case 3 . Focal reactivity for S-100 protein (immunohistochemistry reaction for S-100, original magnification x20). (c) Case 4. Basaloid epithelial cells which vary from small dark cells to larger paler stained cells separated from septa with a solid pattern and small areas of squamous cells with keratinization (hematoxylineosin stain, original magnification x20). (d) Case 4. Consistent and diffuse expression with P63 (immunohistochemistry reaction for P63, original magnification $\mathrm{x} 20$ )

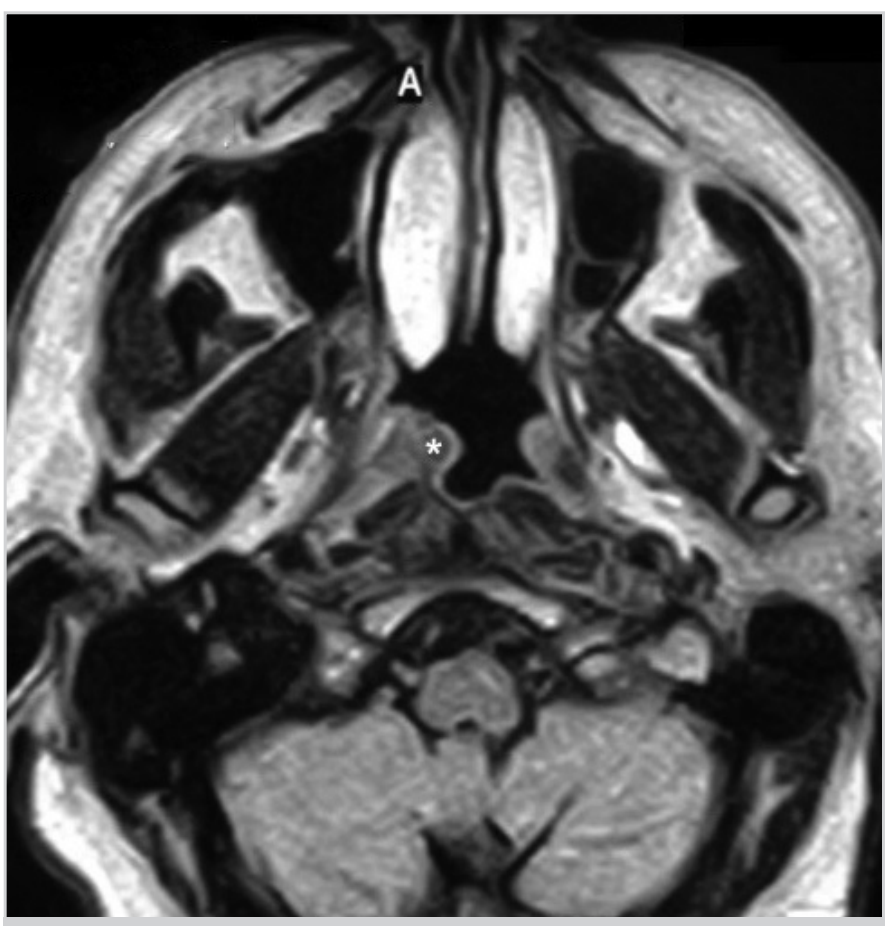

Figure 2. Postcontrast T1 weighted axial image showing a right heterogeneous and irregularly enhanced nasopharyngeal lesion (asterisk)
4) and a complete "en bloc" resection of the lesion was achieved without perioperative complications. The final histologic report showed a benign lesion compatible with pleomorphic adenoma with a prevailing eosinophilic component. No evidence of the disease has been observed in the two years after the surgery.

The mean age of the five patients was 62.2 years (range, 50 to 75 years). Two of the patients were female. The most frequent symptom was unilateral/bilateral nasal obstruction (3/5), epistaxis was present in two patients, while epiphora and hearing loss were observed in one patient each. The primary site of origin of the PA was the lateral nasal wall in three cases, while septum and Rosenmüller's fossa were the origin in one case each. Three lesions were on the left side, one was on the right side and one was on the midline. The type of the endoscopic surgical resection was based on the site of origin and on the extension of the tumor to adjacent sites. All cases were resected "en bloc" by means of endoscopic visualization (Table 1).

No intraoperative complications were observed. In one patient, however, follow-up CT scan three years after the surgery revealed a mucocele in the frontal sinus. A successful endoscopic marsupialization was performed. Endonasal dacryocystorhinostomy was 

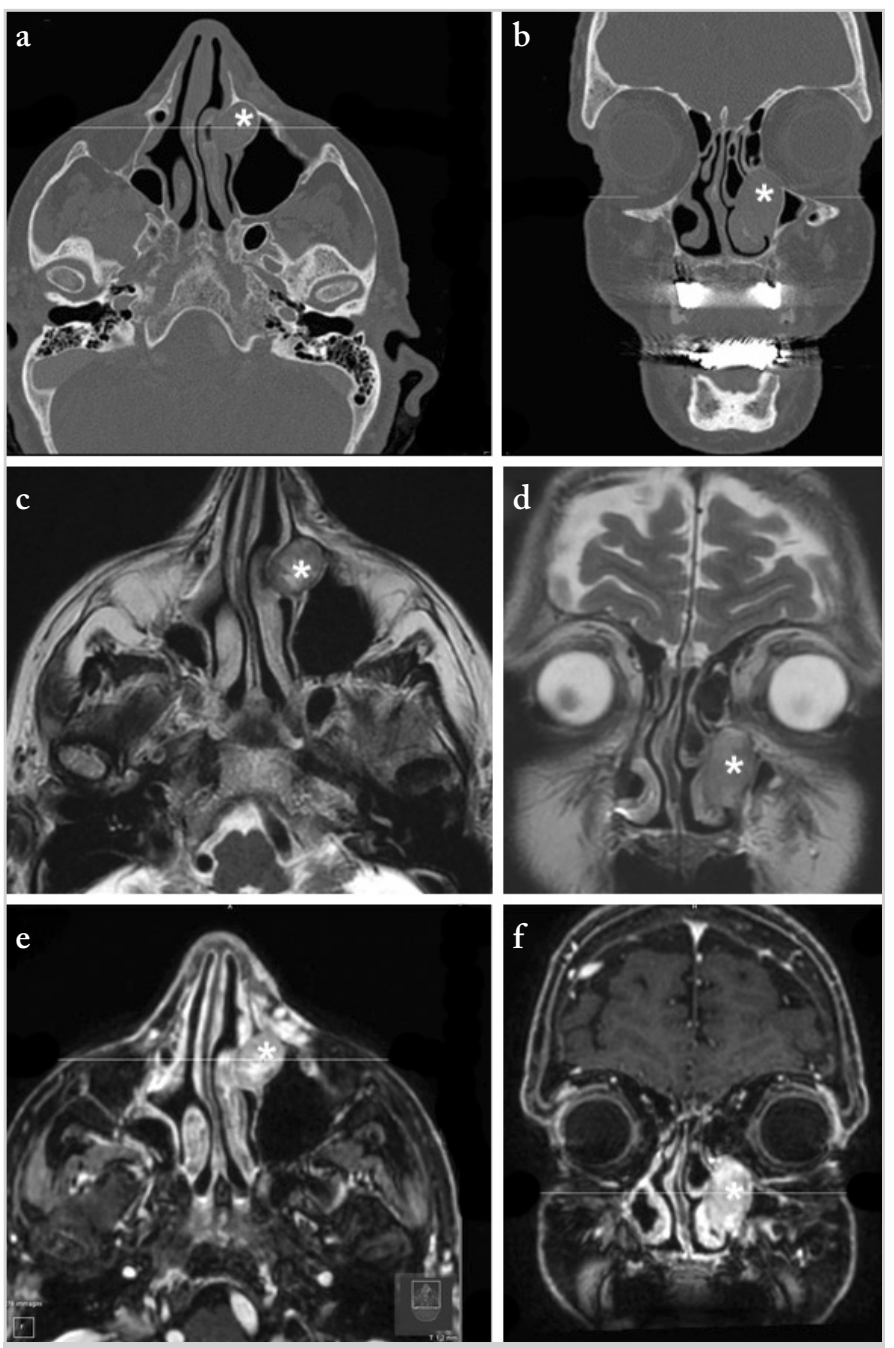

Figure 3. a-f. Preoperative imaging of Case 5. (a, b) CT scan shows swelling of the left nasolacrimal duct with displacement of the bony walls but no signs of erosion or infiltration. (c, d) MR imaging shows a well-circumscribed ovoid lesion of the left nasolacrimal duct with mixed signal in T2-weighted sequences and (e, f) heterogeneous contrast-enhancement in gadolinium-enhanced T1-weighted sequences (asterisks)

performed to another patient who developed a post-saccal lacrimal pathway obstruction one year after the surgery.

None of the patients had recurrence in the mean follow-up period of 127.4 months (range, 25 to 216 months). None of the cases showed malignant transformation into carcinoma ex-PA.

\section{Discussion}

Intranasal PA was first described by Denker and Kahler (5) in 1929, but the first large series of 40 patients with PA of the nasal cavity was not reported until 1973 (6). Since then, multiple single case reports and only one more large series were described in the literature $(2,7)$. The two largest series of intranasal PA include 40 cases by Spiro et al. (5) and 40 cases by Compagno and Wong (7). In the 90s Wakami et al. (8) reviewed 59 cases from the Japanese literature (41 of these cases were previously reported by Suzuki et al. [9]) while Vento et al. (10) recently presented a nationwide series of 10 cases in Finland. According to these studies, majority of the tumors occur in the third to sixth decades of life with a slight female predominance and the nasal septum is the most frequent site of origin, even though the vast majority of minor mucous and serous glands are located in the lateral nasal wall. Nasopharyngeal pleomorphic adenomas are even rarer than the nasal ones and the mainstay of treatment is surgical (3). In our series of PA, there was a slight site predilection for the medial wall of the maxillary sinus (3/5 patients). One out of the five patients had tumor originating from the nasal septum and one from the nasopharynx.

Many theories have been proposed to explain the propensity of intranasal PA to originate from the nasal septum. These historical propositions include: i) the presence of remnants of the vomeronasal organ which is an epithelium-lined duct in the cartilaginous nasal septum degenerated in early fetus; ii) the presence of ectopic embryonic epithelial cells of the nasal septal mucosa, found during the migration of the nasal buds; iii) the direct origin from the mature salivary gland tissue. Viral involvement (polyoma, EBV, CMV, HPV) in the pathogenesis of PA has also been proposed (10). However, the pathogenesis of PA in the sinonasal tract is still unknown. Intriguingly, fewer minor salivary glands are present in the septal submucosa than in other sinonasal anatomic areas (11).

Diagnosis of sinonasal PA may be difficult due to nonspecific symptoms and radiological findings. CT and MRI scans have an important role in treatment planning (Figure 2). Pleomorphic adenoma of the nose generally presents as a well-defined multilobulated soft tissue mass. CT is useful to demonstrate any calcification inside the tumor and any adjacent bony changes (12). MRI findings consist of T1 weighted images low to intermediate signal intensity, T2 weighted images intermediate to high signal intensity and heterogeneous enhancement on contrast enhanced images (13). The pattern of bony alterations, expansive or destructive type changes can help to differentiate benign from malignant lesions. However, these features alone are not sufficient to conclude a definitive diagnosis and histopathological examination is mandatory.

The microscopic aspects of PA of the aerodigestive tract may represent a challenge for the pathologist, particularly in small biopsy samples, in which high cellularity and lack of stromal components may warrant the diagnosis of a malignant neoplasm (7). In these cases, specific expertise in head and neck pathology is required and immunohistochemical stains are useful in reaching the correct diagnosis. In our cases, two biopsies were initially diagnosed as low grade mucoepidermoid carcinoma and extracranial craniopharyngioma, respectively.

Although PA is a biologically benign tumor, it bears a high propensity to recur if is not completely resected. Recurrence rate of intranasal pleomorphic adenomas varies between 5.1\% and $10 \%(2,7,8)$. Consequently, oncological radicality, or resection in clear margins, is the target of the surgery in order to minimize recurrence from residual pseudopodia. This can be obtained through "en bloc" resection as has been emphasized in the treatment of major salivary gland PA (2). In cases of largesized tumors, however, a progressive disassembling of the lesion ("piecemeal" resection) may be employed, keeping in view the 
limits between normal and diseased mucosa, as for the endoscopic transnasal treatment of selected sinonasal and skull base malignancies, may be employed.

Endoscopic techniques have undergone tremendous advancement in the past years. The experience in the management of inflammatory sinonasal pathologies has outlined the opportunity to manage lesions of the skull base and even selected intracranial diseases. The endonasal endoscopic treatment of benign lesions of the sinonasal tract is nowadays well accepted and in the last 10 years, this surgical approach has emerged as a valid treatment option in selected malignant tumors. Absence of external scars and osteotomies, decreased blood loss and post-operative pain, as well as reduced time of hospitalization are the advantages obtained with these techniques. Intranasal PA can be managed by means of endonasal endoscopic surgery using different techniques and approaches (endoscopic septectomy, maxillectomy, centripetal ethmoidectomy, etc.) tailored according to the site of origin of the tumor. According to a recent multivariate analysis, the size of the lesion is not a significant predictor of recurrence, whereas the location of the mass and its relationship with the adjacent paranasal sinuses seem to have a greater impact on the surgical approach (2). In our experience, all five cases were treated through an exclusive endoscopic endonasal "en bloc" resection with no evidence of recurrence after long-term follow-up even in the three cases with involvement of the medial wall of the maxillary sinus.

In the recent years, the experience accumulated by several authors has made possible also the endoscopic transnasal management of PAs of the nasopharynx. The feasibility of the surgical technique is still under evaluation, although data from the literature have become more encouraging year after year, showing no recurrences in the nine endoscopic nasopharyngeal PA cases reviewed by Bowman et al. (3) in 2019. In our case series, we treated one patient with a type 1 nasopharyngeal resection, preoperatively diagnosed as extracranial craniopharyngioma and with a final pathologic diagnosis of PA of the nasopharynx. The patient was followed endoscopically and radiologically for two years with no evidence of recurrence.

In 1990 Freeman et al. (14) reported the only case of intranasal PA with submandibular lymph node metastasis in the literature. However, both the metastatic and the primary lesion appeared benign microscopically. Rarely, PA can also behave in a highly malignant manner, the most common variants being carcinoma ex PA. No case presented malignant transformation or any metastasis in our study.

All patients must undergo a long-term endoscopic follow-up, since PA can recur years after the primary treatment. In case of a suspicious lesion or in the presence of abundant scar tissue obstructing the site of origin of the mass, imaging (MRI with gadolinium contrast) and subsequent biopsies must be performed.

\section{Conclusion}

PA of the nasal cavities is an uncommon, benign tumor presenting endoscopically as a midline, bilateral or a unilateral lesion. CT scan and/or MRI with contrast enhancement are mandatory in order to define the lesion's extension and vascular morphology. Biopsies are useful when the tumor has low blood supply and when meningoencephalocele has previously been excluded. PAs of the sinonasal tract and the nasopharynx are difficult to diagnose because of nonspecific clinical and radiological findings. In fact, an accurate histopathological examination by an expert head and neck pathologist is needed to avoid a preoperative misdiagnosis as sinonasal malignancies due to the challenging microscopical picture of some cases and the small size of the sample. This should be avoided, as it leads to an unnecessary overtreatment. Endoscopic endonasal approaches can be considered as the first choice for the treatment of these tumors with excellent visual control of the surgical field and clear margins.

Ethics Committee Approval: Ethics committee approval was received from the Board of Ethics of Ospedale di Circolo e Fondazione Machi (Approval Date: July 7, 2015; Approval Number: 0033025).

Informed Consent: Informed consent was obtained from the patients.

Peer-review: Externally peer-reviewed.

Author Contributions: Concept - A.K., G.B., G.P., P.B., M.T.Z., G.O., M.D.L.; Design - A.K., G.P.; Supervision - A.K.; Resources G.B.; Data Collection and/or Processing - G.B.; Analysis and/or Interpretation - P.B., M.T.Z., F.C., M.A.; Literature Search - P.B., M.T.Z., F.C., M.A., M.D.L., S.U., P.C.; Writing - G.P., G.O., S.U., P.C.; Critical Reviews - F.C., M.A., G.O., M.D.L., S.U., P.C.

Conflict of Interest: The authors have no conflicts of interest to declare.

Financial Disclosure: The authors declared that this study has received no financial support.

\section{References}

1. El-Naggar AK, Chan JKC, Grandis JR, Takata T, Slootweg PJ, editors. WHO Classification of Head and Neck Tumours. Lyon, France: IARC; 2017.

2. Rha MS, Jeong S, Cho HJ, Yoon JH, Kim CH. Sinonasal pleomorphic adenoma: a single institution case series combined with a comprehensive review of literatures. Auris Nasus Larynx 2019; 46: 223-9. [Crossref]

3. Bowman J, Daudia A, Sahasrabudhe N, Belloso A. Coblator-assisted endoscopic transnasal resection of a large nasopharyngeal pleomorphic adenoma. Case Rep Otolaryngol 2019; 2019: 4654357. [Crossref]

4. Castelnuovo P, Nicolai P, Turri-Zanoni M, Battaglia P, Bolzoni Villaret A, Gallo S, et al. Endoscopic endonasal nasopharyngectomy in selected cancers. Otolaryngol Head Neck Surg 2013; 149: 424-30. [Crossref]

5. Denker A, Kahler O, editors. Handbuch der Hals, Nasen und Ohrenheilkunde. Berlin: Springer, 1929; 5: 202.

6. Spiro RH, Koss LG, Hajdu SI, Strong EW. Tumors of minor salivary origin. A clinicopathologic study of 492 cases. Cancer 1973; 31: 117-29. [Crossref] 
7. Compagno J, Wong RT. Intranasal mixed tumors (pleomorphic adenomas): a clinicopathologic study of 40 cases. Am J Clin Pathol 1977; 68: 213-8. [Crossref]

8. Wakami S, Muraoka M, Nakai Y. [Two cases of pleomorphic adenoma of the nasal cavity]. Nihon Jibiinkoka Gakkai Kaiho 1996; 99: 38-45. [Crossref]

9. Suzuki K, Moribe K, Baba S. [A rare case of pleomorphic adenoma of lateral wall of nasal cavity. With special reference of statistical observation of pleomorphic adenoma of nasal cavity in Japan]. Nihon Jibiinkoka Gakkai Kaiho 1990; 93: 740-5. [Crossref]

10. Vento SI, Numminen J, Kinnunen I, Rautiainen M, Tarkkanen J, Hagström J, et al. Pleomorphic adenoma in the nasal cavity: a clinicopathological study of ten cases in Finland. Eur Arch Otorhinolaryngol 2016; 273: 3741-5. [Crossref]

11. Wallace RD, Arendt DM, Irene RT. Pathologic quiz case 1. Pleomorphic adenoma. Arch Otolaryngol Head Neck Surg 1990; 116: 486-8.

12. Oztürk E, Sağlam O, Sönmez G, Cüce F, Haholu A. CT and MRI of an unusual intranasal mass: pleomorphic adenoma. Diagn Interv Radiol 2008; 14: 186-8.

13. Motoori K, Takano H, Nakano K, Yamamoto S, Ueda T, Ikeda M. Pleomorphic adenoma of the nasal septum: MR features. AJNR Am J Neuroradiol 2000; 21: 1948-50.

14. Freeman SB, Kennedy KS, Parker GS, Tatum SA. Metastasizing pleomorphic adenoma of the nasal septum. Arch Otolaryngol Head Neck Surg 1990; 116: 1331-3. [Crossref] 